\title{
Cálculo de la distribución de voltajes en las cadenas de aisladores de una línea de transmisión de 500 kV utilizando el método de los elementos finitos
}

\section{(Calculation of Voltage Distribution along the Insulator Strings of a $500 \mathrm{kV}$ Transmission Line Based on Finite Element Method)}

\author{
Juan Ramírez"1, Krissia Cabezas ${ }^{1}$, Pedro Jiménez ${ }^{1}$, Raúl Canelos ${ }^{1}$, Bolívar Escobar ${ }^{1}$
}

\begin{abstract}
Resumen
En este artículo se puede apreciar las distribuciones de voltaje a lo largo de una cadena de aisladores de una línea de transmisión de 500 kV de Ecuador. El problema electrostático relacionado a la distribución de voltajes se resolvió utilizando el método de los elementos finitos. Se realiza un dibujo a escala 1:1 en una herramienta de dibujo tipo CAD para luego ser exportada al programa FEMM, que resuelve el problema y del que se obtienen los resultados relacionados a los voltajes en la cadena de aisladores. Se aprecia las diferencias de voltaje en los diferentes aisladores que componen una cadena y se observa la diferencia entre las cadenas, determinada por la ubicación geométrica en el espacio de solución.
\end{abstract}

\section{Palabras clave}

Aisladores de suspensión de alto voltaje; aislamiento eléctrico; distribución de voltaje en la cadena de aisladores; FEMM; método de los elementos finitos.

\begin{abstract}
This paper presents the voltage distributions along the insulator strings of a $500 \mathrm{kV}$ overhead transmission line of Ecuador. The electrostatic problem related to the voltage distribution was solved using the Finite Element Method. A 1: 1 scale drawing is done on a CAD type drawing tool and then exported to the FEMM program that solves the problem and the results related to the voltages in the insulator string are obtained. The voltage differences in the different insulators that constitute a string are appreciated and the difference between the strings determined by the geometric location in the solution space is observed
\end{abstract}

\section{Keywords}

High voltage suspension insulators; electric insulation; voltage distribution along the Insulator strings, FEMM, Finite Element Method.

\section{Introducción}

Las líneas de transmisión de alto voltaje permiten transportar la energía eléctrica a grandes distancias, de una manera eficiente y segura, desde los centros de generación hacia los centros de consumo. En los últimos años, con los avances tecnológicos, la incorporación de grandes cargas al sistema y el cambio de la matriz productiva, el Ecuador ha tenido un crecimiento tendencial del consumo de energía eléctrica. Según los estudios realizados por la Agencia de Regulación y Control de la Electricidad del Ecuador (Arconel), el crecimiento promedio del consumo de energía en el país es del 5 \% anual, como se presenta en la figura 1 (Arconel, 2013), siendo esta una razón para la construcción de uno de los proyectos hidroeléctricos más grandes en el país: Coca Codo Sinclair, con una capacidad instalada de 1500 MW. 
Con el fin de evacuar la generación de energía eléctrica de esta central, se estableció como mejor alternativa la implementación de un sistema de transmisión de 500 kV de extra alto voltaje que, además de vincular la nueva central hidroeléctrica Coca Codo Sinclair con Quito, permitirá interconectarla con el principal centro de carga del país en Guayaquil (Conelec, 2013; Transelectric, 2015).

Figura 1. Evolución histórica y proyección del consumo de energía en el Ecuador hasta el año 2022. Fuente: Plan Maestro de Electrificación 2013-2022 del Ecuador (Arconel, 2013)

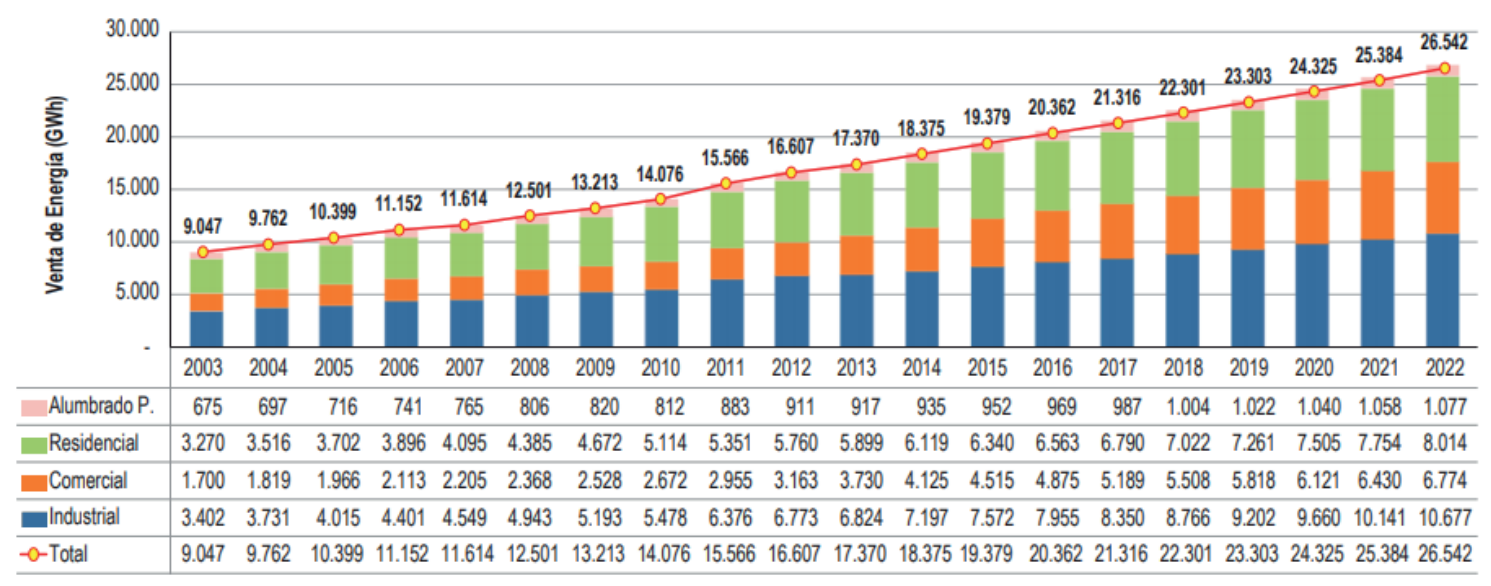

El sistema de 500 kV está compuesto por una línea de transmisión con dos circuitos en paralelo que parten, desde la subestación del proyecto hidroeléctrico Coca Codo Sinclair ubicada en el cantón El Chaco, provincia de Napo, y finalizan en la subestación El Inga situada en el cantón Quito, provincia de Pichincha, con una longitud total de 126 km cada línea.

Las rutas de las líneas atraviesan zonas de altitudes que varían entre 1200 m.s.n.m. en el sector del proyecto de generación Coca Codo Sinclair, y 4100 m.s.n.m. en el sector más alto conocido como el Paso de la Virgen. Cada una de las fases de la línea de transmisión está constituida por un haz de 4 conductores de aleación de aluminio ACAR 1100 MCM 18/19, con dos cables de guarda cada una. Los conductores se sostienen en estructuras metálicas de celosía, autosoportantes, de tipo "cabeza de gato" y configuración horizontal. Las cadenas de aisladores en suspensión son en "V" o "I", en cada una de las fases, y son de porcelana o vidrio templado (Transeselectric, 2013).

Con el incremento de los niveles de voltaje en los sistemas de transmisión, los materiales aislantes soportan campos eléctricos muy intensos, por lo que para su diseño resulta muy importante conocer la distribución de las líneas de campo eléctrico y líneas equipotenciales a lo largo de su geometría. De esta manera se puede verificar el comportamiento del material, cuando está sometido a los altos voltajes (Khare, Hasabe y Mandlik, 2016). En nuestro país, en la línea de extra alto voltaje de $500 \mathrm{kV}$, es evidente que la incidencia de los campos electromagnéticos será más intensa, ya que a mayor nivel de voltaje, mayor es la intensidad de los campos electromagnéticos (Radwan, Mahdy, Abdel-Salam y Samy, 2013).

En la actualidad, el desarrollo de programas que utilizan métodos de elementos finitos brinda la facilidad de resolver gráficamente las situaciones relacionadas con los problemas electromagnéticos; por ejemplo: el programa computacional FEMM (Finite Element Method Magnetics) que sobre la base de métodos de elementos finitos FEM permite resolver las ecuaciones diferenciales de Maxwell, implícitas en los temas de campos eléctricos y magnéticos. 
Es así como, para diferentes configuraciones de líneas de transmisión, se puede obtener las soluciones de los campos electromagnéticos, bastante aproximadas a las reales.

Se han puesto a prueba varios modelos numéricos basados en las soluciones de las ecuaciones diferenciales parciales, mediante el método de los elementos finitos en los aisladores, para determinar las distribuciones de voltaje y campos eléctricos en ellos (Bessedik, 2015; Khare et al., 2016), que se realizan con consideraciones simples de la geometría de las líneas y buscan obtener una distribución de voltajes más uniforme (Huang, Ruan y Liu, 2010; Zhang, He, Zeng y Liang, 2010).

El método de elementos finitos FEM presenta buenos resultados en el cálculo del campo eléctrico y la distribución de potenciales en los aisladores de alto voltaje, y es uno de los métodos numéricos más exitosos en el momento de resolver problemas electrostáticos que incluso consideran la contaminación (Sima, Yuan, Yang, et al., 2010). Además, es un método bastante flexible ya que permite resolver las ecuaciones asociadas a los campos electromagnéticos con técnicas sencillas y gráficas, para estimar el valor de los campos en las superficies de los electrodos de diferentes materiales dieléctricos. Por último, se adapta bien a diferentes configuraciones de geometría (Arora y Mosch, 2011; Benguesmia, M'Ziou y Boubakeur, 2018).

El conocimiento de la distribución de voltajes a lo largo de una cadena de aisladores en una torre de transmisión, permite verificar que los aisladores no estén sometidos a voltajes que pueden llegar a esforzarlos dieléctricamente más de lo que requieren; esto, debido a que en la cadena de aisladores el voltaje no se distribuye uniformemente sino que por lo general el o los primeros aisladores cercanos al conductor soportan más voltaje que los aisladores que están sujetos a las estructuras de metal de las torres, por eso, usualmente los aisladores más cercanos sufren mayores esfuerzos dieléctricos y tienen una mayor probabilidad de sufrir una falla de aislamiento eléctrico. La distribución de voltajes en la cadena de aisladores se ve afectada por las condiciones geométricas de los aisladores y de las estructuras en las que se instalan (Du Zhiye y Daochun, 2010).

El estudio realizado por Antonov, Glushkov y Kropotuhin (2016), utiliza el método FEM para resolver las distribuciones de voltaje en cadenas de aisladores, apoyándose en experimentos de laboratorio hasta niveles de $150 \mathrm{kV}$. El estudio determina que las desviaciones entre el modelo resuelto con FEM y los experimentos van desde un $3.7 \%$ a un $11.2 \%$. Además, encuentran que los aisladores que están aproximadamente a un tercio de la longitud de la cadena, desde la cruceta de la torre de transmisión, son los que menos voltaje soportan. El estudio no llega a voltajes más altos por las limitaciones de sus laboratorios.

En el artículo de Ashouri, Mirzaie y Gholami (2010), se realiza una solución con el método FEM en una cadena de aisladores de $230 \mathrm{kV}$. Pudieron determinar que el incremento del tamaño de la cadena de aisladores permite tener una distribución de voltajes más uniforme. Por su parte, en el estudio de Akbari, Mirzaie, Rahimnejad y Asadpoor (2012), se observa el efecto de un anillo metálico en el final de una cadena de aisladores de $230 \mathrm{kV}$, resolviendo el problema con el método FEM, y se observa que este anillo puede mejorar las distribuciones de voltaje en la cadena, pero debe optimizarse de acuerdo con las características particulares de la cadena en estudio. Se indica también que alargar la cadena hace más uniforme la distribución de voltajes.

La medición de la distribución de voltajes en la cadena de aisladores es difícil de realizar a medida que el voltaje es mayor. La disponibilidad de instrumentos adecuados o de laboratorios con las capacidades de medición y generación de altos voltajes limita la posibilidad de realizar la medición de estas distribuciones y es más práctico recurrir a paquetes de simulación.

Con estos antecedentes, en este artículo se propone como objetivo realizar el cálculo de la distribución de voltajes en la cadena de aisladores de una torre de transmisión de 500 kV 
de la línea San Rafael - El Inga de Ecuador, la cual tiene dos cadenas de suspensión: una en I y una en V. Para poder obtener la solución se recurre al método FEM con el software FEMM, para realizar los cálculos en un plano bidimensional 2-D.

En la sección 2 del este artículo se presenta una base teórica del estudio a realizar. En la sección 3 se presenta la metodología propuesta y las descripciones de los materiales y geometría a utilizar. La sección 4 presenta los resultados obtenidos y su discusión. Finalmente en la sección 5 se encuentran las conclusiones del estudio realizado

\section{Problema electrostático}

El problema del cálculo de los campos eléctricos en un aislador está dentro de la categoría de problemas electrostáticos en la ingeniería (Khare et al., 2016). Estos problemas consideran el comportamiento de la intensidad de campo eléctrico $E$ y la densidad de flujo eléctrico $D$. Tales cantidades deben cumplir con la forma diferencial de la ley de Gauss, la cual enuncia que el flujo que sale de un volumen cerrado es igual a la carga contenida en ese volumen (Meeker, 2006; Muniraj y Chandrasekar, 2012). Las ecuaciones básicas utilizadas para calcular los potenciales $\checkmark$ y el campo eléctrico son las leyes de Maxwell, que para el modelo electrostático están presentadas en las ecuaciones (1), (2) y (3):

$$
\begin{gathered}
\operatorname{div} \vec{D}=\rho \\
\vec{D}=\varepsilon \vec{E} \\
\vec{E}=-\vec{\nabla} V
\end{gathered}
$$

Donde $D$ es la densidad de flujo eléctrico, $E$ es la intensidad de campo eléctrico, $\rho$ es la densidad de carga eléctrica superficial, $\varepsilon$ es la permitividad del material dieléctrico y $V$ es el potencial eléctrico.

La combinación de las ecuaciones (1), (2) y (3) resultan en la ecuación (4):

$$
\operatorname{div} \varepsilon(-\vec{\nabla} V)=\rho
$$

La ecuación (4) se conoce como ecuación de Poisson. La ecuación de Laplace se puede obtener haciendo $\rho=0$. En el caso del equipamiento de alto voltaje, las cargas espaciales no están presentes o son despreciables $(\rho=0)$, por lo que se debe resolver la ecuación (5) (Nicolopoulou et al., 2011).

$$
\operatorname{div} \varepsilon(-\vec{\nabla} V)=0
$$

La aplicación de la ley de Gauss lleva a la ecuación (5) a constituirse en una ecuación diferencial parcial de segundo orden, dada en (6) o (7):

$$
\begin{gathered}
-\varepsilon \nabla^{2} V=0 \\
-\varepsilon\left(\frac{\partial^{2} V}{\partial x^{2}}+\frac{\partial^{2} V}{\partial y^{2}}+\frac{\partial^{2} V}{\partial z^{2}}\right)=0
\end{gathered}
$$


El programa FEMM resuelve internamente las ecuaciones (6) o (7) para el potencial $V$ sobre un dominio discreto, con fuentes y condiciones definidas por el usuario. FEMM realiza una discretización espacial de las ecuaciones diferenciales creando pequeños elementos de forma triangular. De esa manera pasa de un problema pequeño pero muy difícil de solucionar, a un problema muy grande pero fácil de resolver, pues se conocen las soluciones aproximadas en las cercanías de los elementos, dadas por las condiciones de borde (Meeker D., 2006).

Un problema en una cadena de aisladores de suspensión con aisladores idénticos de tipo disco es la distribución del voltaje a lo largo de la cadena. Cada aislador se convierte en un capacitor y se tiene como resultado que el voltaje en cada aislador no es el mismo. El voltaje en los aisladores cambia según su ubicación y el máximo gradiente se da en el aislador más cercano al conductor (Wadhwa, 1989).

Existen diferentes métodos de solución para determinar la distribución de voltajes en una cadena de aisladores basados en métodos de simulación de carga, modelos capacitivos y elementos finitos (Chen y Liang, 2012). En este documento se modela a la cadena de aisladores para ser utilizada en el software FEMM y utilizar un método FEM, pues es de libre acceso y presenta facilidad en la representación geométrica de la línea en estudio.

\section{Metodología}

Se utilizó como modelo la geometría de una torre de transmisión de 500 kV trifásica típica de la Línea de Transmisión San Rafael-El Inga, que lleva la energía producida por la Central Hidroeléctrica Coca Codo Sinclair a la Subestación de El Inga en la ciudad de Quito-Ecuador. La torre se puede apreciar de manera simplificada en la figura 2 (Transeselectric, 2013). La torre tiene 3 cadenas de aisladores (dos en tipo I y una en tipo V). Hay 4 conductores por fase con conductores ACAR 1100 MCM separados $45 \mathrm{~cm}$ en el haz de conductores. Los dos conductores de guardia fueron ignorados en el estudio.

Figura 2. Geometría simplificada y dimensiones mayores de la torre de transmisión de suspensión de la línea de 500 kV, unidades en m.

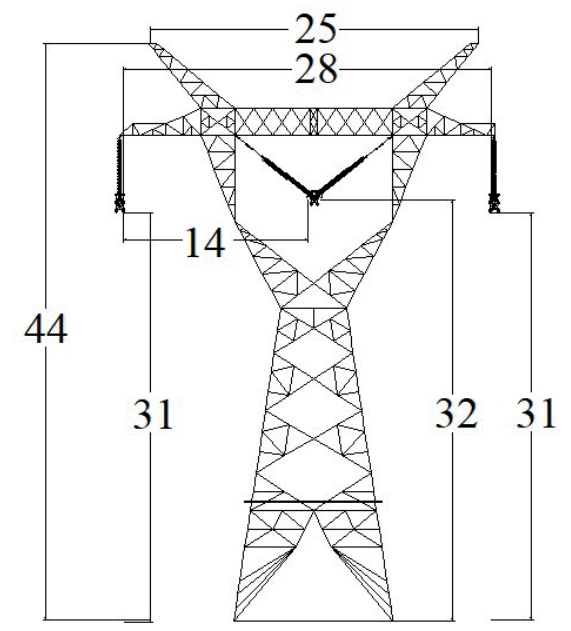

La cadena de aisladores está conformada por 28 aisladores de suspensión de porcelana tipo U300BP según la norma IEC60305, cuyas características se presentan en la figura 3. 
Figura 3. Aislador U300 BP utilizado en el estudio

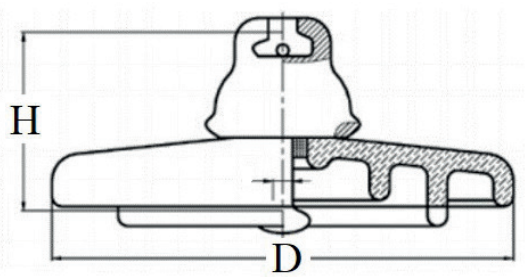

El aislador tiene un diámetro D de 340 mm, un espaciamiento H de 195 mm, una distancia de fuga de $550 \mathrm{~mm}$. El voltaje de perforación del aislamiento es de $130 \mathrm{kV}$. El nivel de aislamiento de frecuencia industrial en seco es de $90 \mathrm{kV}$. El nivel de aislamiento de frecuencia industrial en mojado es de 55 kV y el nivel de aislamiento ante impulso CFO positivo y negativo es de 130 kV.

Figura 4. Diagrama de flujo para la obtención de voltajes con FEMM

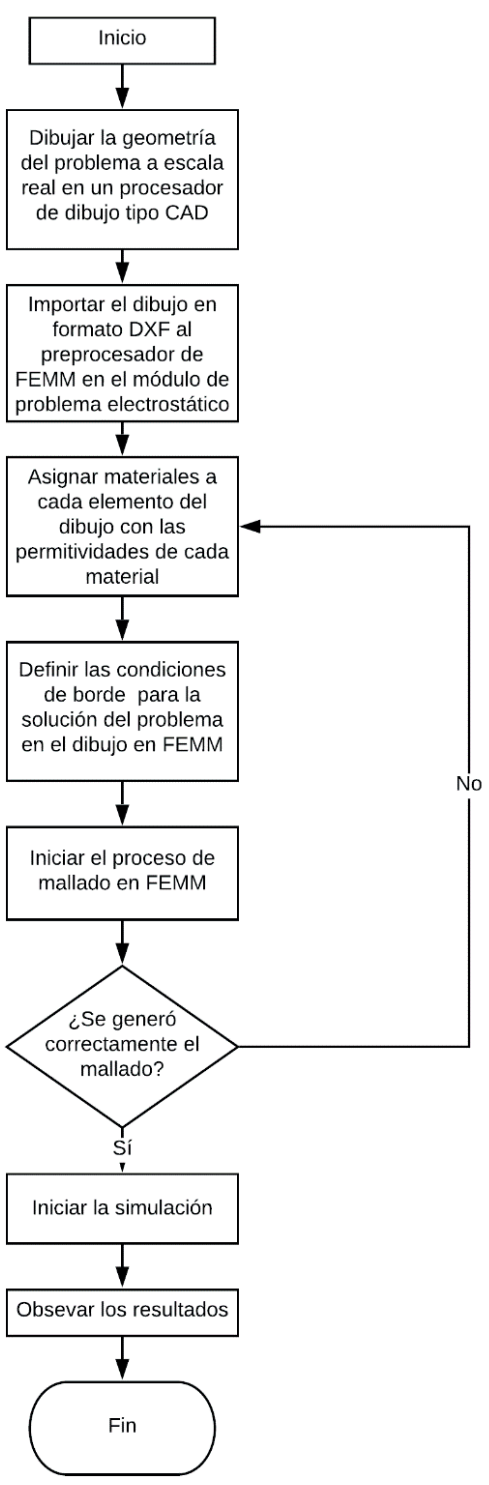


En la Figura 4 se presenta un diagrama de flujo del proceso de simulación que se sigue para obtener las distribuciones de voltaje en este artículo. Para la simulación de esta torre en FEMM, se realiza en primer lugar un dibujo a escala real en un editor de dibujo tipo CAD, que luego se importa al preprocesador de FEMM. Una vez en FEMM se deben atribuir los parámetros de los materiales a utilizar; estos valores están dados en la tabla 1 (Ordal et al., 1982).

Se definen las condiciones de borde de los conductores según los voltajes de fase más altos del sistema; para ello se considera el voltaje pico que corresponde a la peor condición de 528 kV rms del voltaje de línea. Y la condición de borde de voltaje igual a cero en el suelo y a una distancia de $35 \mathrm{~m}$ a cada lado del eje de la torre.

Luego se generan los triángulos de los elementos finitos en el software y se procede a la simulación, donde se puede obtener los resultados de campo eléctrico y potenciales en el espacio.

Tabla 1. Propiedades de los materiales para la simulación con FEMM. (Fuente: Ordal et al., 1982)

\begin{tabular}{|l|c|}
\hline \multicolumn{1}{|c|}{ Material } & Permitividad relativa $\varepsilon \mathbf{r}$ \\
\hline Acero galvanizado & 500000 \\
\hline Cemento & 18 \\
\hline Porcelana & 5.9 \\
\hline Aluminio & 50000 \\
\hline Hierro fundido & 500000 \\
\hline Aire & 1 \\
\hline Suelo húmedo & 30 \\
\hline Suelo seco & 15 \\
\hline Suelo muy seco & 3 \\
\hline
\end{tabular}

El modelo realizado en FEMM puede encontrarse en la dirección https://github.com/juandave1988/FEMM.git y ha sido subido por los autores para utilidad de la comunidad relacionada.

\section{Resultados y discusión}

En primer lugar, se creó un aislador que se presenta en el preprocesador de FEMM en la figura 5a. Para probar el modelo del aislador se realiza la simulación a 90 kV, que es el máximo voltaje de frecuencia industrial especificado por la norma para el aislador de la figura 3. La simulación resultante se aprecia en la figura 5b.

Figura 5. (a) Aislador U300 BP mallado en FEMM.

(b) Resultados de la simulación FEM en el Aislador U300 BP en FEMM

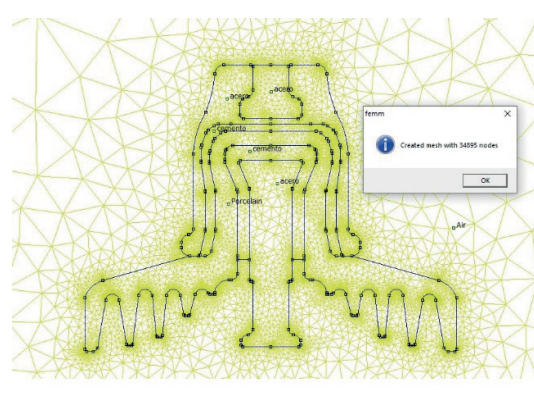

(a)

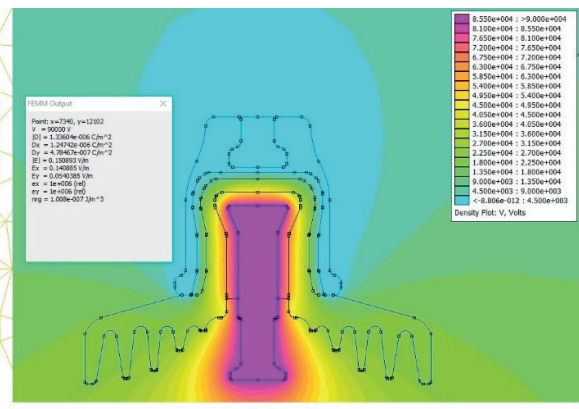

(b) 
En la figura 5b se puede apreciar la distribución de potenciales como un mapa de densidades en colores. El color violeta presenta un potencial de $90 \mathrm{kV}$ y el celeste el potencial de $0 \mathrm{kV}$. Se puede apreciar cómo cambian los potenciales según los colores en el espacio y con la herramienta de medición del software es posible conocer el valor del campo eléctrico, y potencial, en cualquier punto de este espacio simulado.

Una vez puesto a prueba el modelo del aislador se procede con el modelo completo de la línea con todos sus herrajes y aisladores. En las figuras 6a, 6b y 6c, se presentan los modelos en el preprocesador de FEMM.

Figura 6. (a) Torre de transmisión modelada en FEMM.

(b) Acercamiento a la cadena de aisladores de suspensión tipo I modelada en FEMM.

(c) Acercamiento a la cadena de aisladores de suspensión tipo V modelada en FEMM

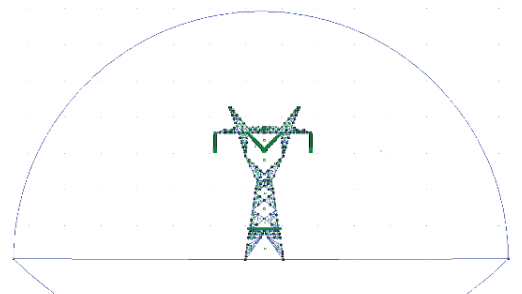

(a)

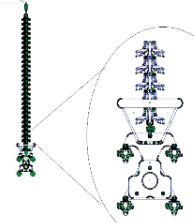

(b)

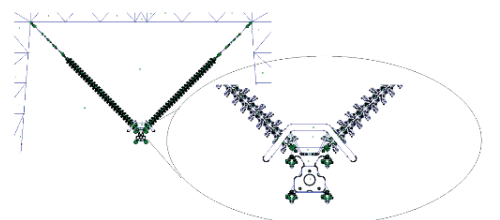

(c)

Una vez que se procede a la simulación, se obtiene los resultados que gráficamente pueden apreciarse en las figuras $7^{\mathrm{a}}, 7 \mathrm{~b}$ y $7 \mathrm{c}$.

Figura 7. (a) Resultados de la simulación FEM en la torre de transmisión modelada en FEMM.

(b) Acercamiento a los resultados de la cadena de suspensión tipo $V$ de la torre de transmisión

modelada en FEMM. (c) Acercamiento a los resultados de la cadena de suspensión tipo I de la torre de transmisión modelada en FEMM

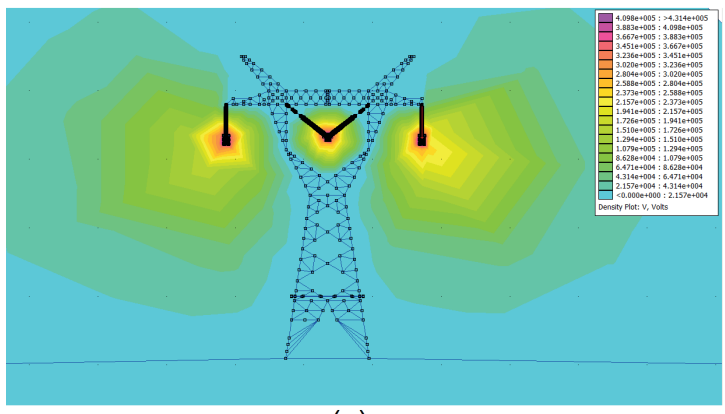

(a)

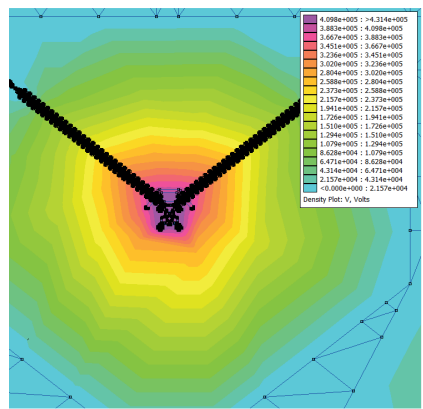

(b)

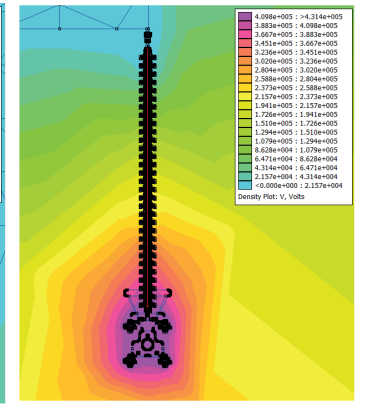

(c)

A pesar de que FEMM posee una herramienta para realizar gráficos llamada PLOT, para graficar el potencial a lo largo de una línea, que en este caso serían las cadenas de aisladores, 
se recurre a un script de Python 3.7, el cual extrae los resultados en un archivo de texto plano de los sitios que se desee en la simulación, para luego tratar a estos resultados de mejor manera (Meeker D., 2018). Con los valores de potencial obtenidos de la simulación y luego de ser procesados, se obtiene los voltajes a lo largo de las cadenas de aisladores, en las figuras 8a, 8b, 8c y $8 d$, y en la tabla 2, siendo el aislador 1 el más cercano al conductor.

Las figuras $8 \mathrm{a}, 8 \mathrm{~b}, 8 \mathrm{c}$ y $8 \mathrm{~d}$ presentan la distribución de voltaje a lo largo de la cadena de aisladores de la tabla 2. La longitud de la cadena está expresada en porcentaje, siendo el nivel 0 $\%$ medido desde el aislador 1 , que es el que sostiene a los conductores energizados; así, el 100 \% sería la longitud de la cadena hasta la cruceta de la torre de transmisión.

En la distribución de voltaje a lo largo de la cadena de aisladores resulta interesante observar que a pesar de que la torre de transmisión fue modelada simétricamente y las cadenas y aisladores son idénticas, la solución numérica indica una diferencia entre las cadenas de suspensión del lado derecho e izquierdo de la torre, como se aprecia en las figuras 8a y 8b. La cadena del lado derecho (8a) presenta cambios de voltajes más pronunciados, mientras que en la cadena izquierda (8b), se tiene una distribución de voltajes más uniforme. Por otra parte, las figuras 8c y $8 d$ de las dos cadenas del brazo central en $V$, se comportan prácticamente iguales y existe una uniformidad en la distribución de voltajes en la cadena, hasta cerca del $90 \%$ de la cadena. En ese punto hay un cambio en la distribución de voltajes que indicaría, que un aislador está soportando más voltaje que el resto de los aisladores de la cadena.

La figura 9. Presenta el voltaje que soporta cada aislador en la cadena, expresado como un porcentaje del voltaje total de la cadena. Se aprecia, de mejor manera en las figuras 9a y 9b, que los aisladores 8, 9, 20 y 25 de la cadena derecha soportan una mayor diferencia de potencial (ddp) que el resto de la cadena, mientras que en la cadena izquierda los aisladores 8, 9, $17,25,26$ y 28 soportan una mayor ddp pero en menor relación respecto al resto de aisladores de esa cadena. Por otra parte, las figuras 9 c y $9 \mathrm{~d}$ de las dos cadenas del brazo central en $\mathrm{V}$, se comportan prácticamente iguales, pero en su aislador número 25 presentan una mayor ddp, que alcanza aproximadamente el 16 \% (aproximadamente $69 \mathrm{kV}$ ), del voltaje de la cadena. Este efecto resulta muy interesante y plantearía la posibilidad de investigar (en un posible trabajo futuro), si se debe o no a un efecto de la geometría, al estar esa cadena "encerrada" en la parte central de la torre.

En la tabla 2 se aprecia que en la mayoría de los aisladores no hay una ddp que suponga problemas para el aislamiento, pues tienen voltajes que van hasta aproximadamente un $4 \%$ (unos $17 \mathrm{kV}$ ). Hay otros aisladores que tienen valores de ddp mayores al $4 \%$, en los que se aprecia que el mayor valor se da en el aislador número 25 de la cadena de suspensión izquierda del brazo en V, con una ddp del 16.06 \% o 69.2 kV. Según las especificaciones del aislador U300BP de la figura 3, este aislador estaría operando con normalidad dentro de los valores especificados por la norma para su diseño en estado seco, que es de 90 kV. Pero en condiciones de humedad, lluvia o contaminación podría producirse una falla de aislamiento en este aislador, ya que el nivel de aislamiento en condiciones de humedad es de $55 \mathrm{kV}$. Sin embargo, para aseverar si este aislador puede fallar se requiere realizar el ensayo de laboratorio que determine su nivel de aislamiento real, pues las especificaciones del aislador son estandarizadas con valores mínimos que no son en realidad los que presenta el aislador, y que por lo general suelen ser superiores ${ }^{2}$. Por otra parte, se debería correr la simulación una gran cantidad de veces, utilizando un método aleatorio,

2 Experiencia del autor en la ejecución de ensayos de aislamiento de frecuencia industrial, en el Laboratorio de Alto Voltaje de la Escuela Politécnica Nacional 
como el de Montecarlo, que modifique las variables que influyen en esta simulación FEM, y con la determinación de esos resultados obtener una probabilidad de que el voltaje en ese aislador sea de cierto valor. Esta actividad es factible de ejecutarse con el modelo implementado en FEMM, pero excede el alcance de este artículo y será retomada en los trabajos futuros.

Figura 8. (a) Distribución del voltaje a lo largo de la cadena de aisladores tipo I. Lado derecho.

(b) Distribución del voltaje a lo largo de la cadena de aisladores tipo I. Lado izquierdo.

(c) Distribución del voltaje a lo largo del brazo izquierdo de la cadena de aisladores tipo V.

(d) Distribución del voltaje a lo largo del brazo derecho la cadena de aisladores tipo V

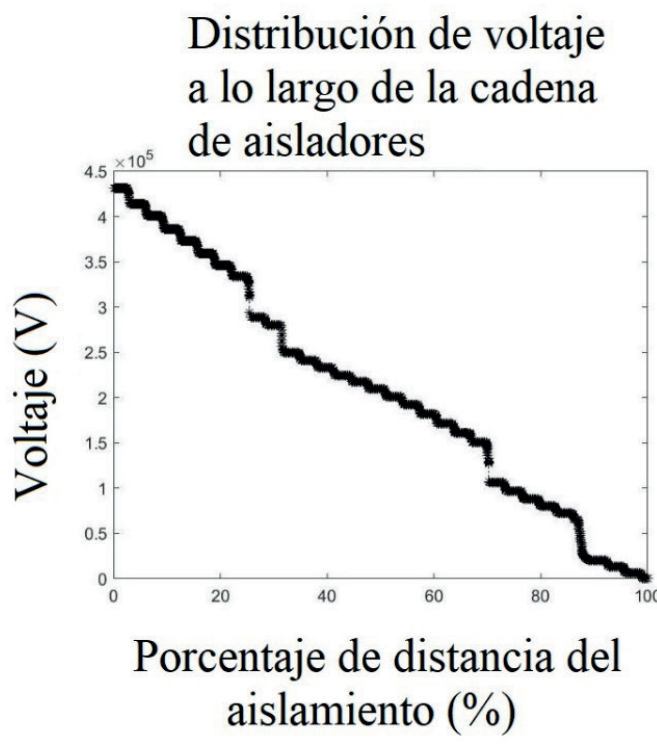

(a)

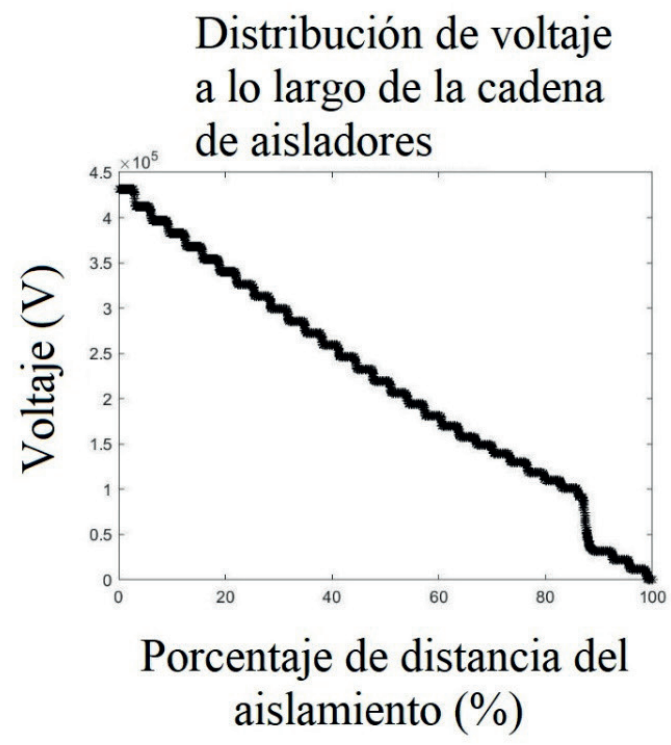

(c)

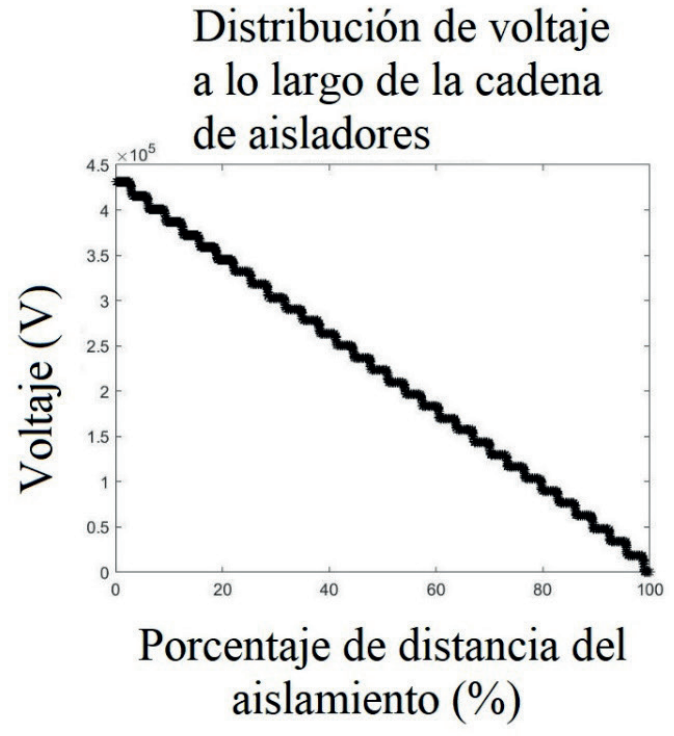

(b)

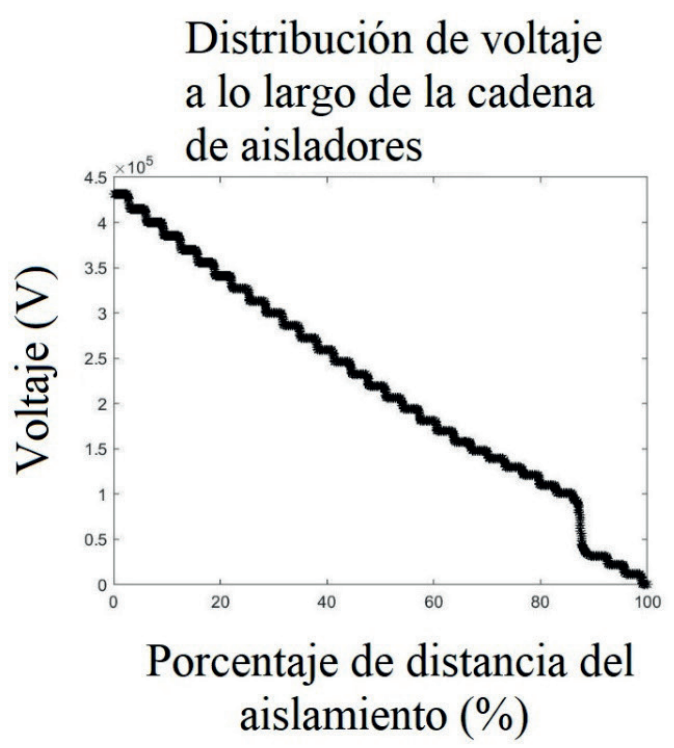

(d) 
Tabla 2. Distribución de voltajes en las cadenas de aisladores como resultado de la simulación con FEMM

\begin{tabular}{|c|c|c|c|c|c|c|c|c|}
\hline 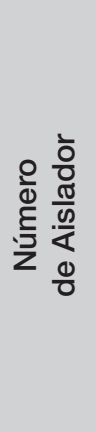 & 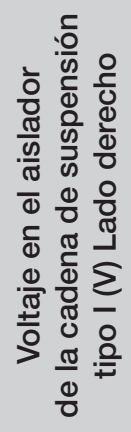 & 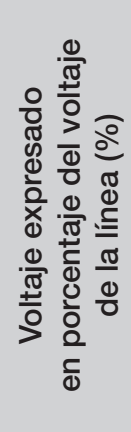 & 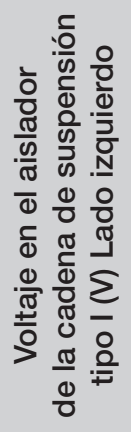 & 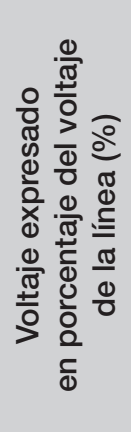 & 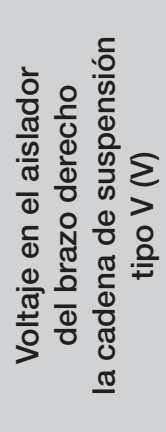 & 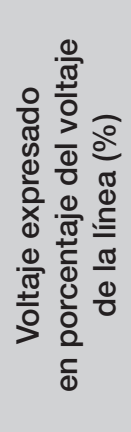 & 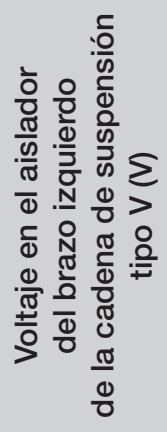 & 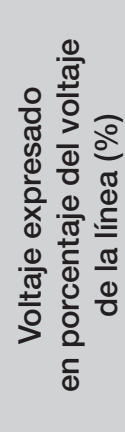 \\
\hline 1 & 17000 & 3.94 & 15000 & 3.48 & 16000 & 3.71 & 19000 & 4.41 \\
\hline 2 & 13000 & 3.02 & 15000 & 3.48 & 15000 & 3.48 & 15000 & 3.48 \\
\hline 3 & 15000 & 3.48 & 14000 & 3.25 & 15000 & 3.48 & 14000 & 3.25 \\
\hline 4 & 13000 & 3.02 & 15000 & 3.48 & 15000 & 3.48 & 15000 & 3.48 \\
\hline 5 & 14000 & 3.25 & 13000 & 3.02 & 14000 & 3.25 & 14000 & 3.25 \\
\hline 6 & 13000 & 3.02 & 14000 & 3.25 & 15000 & 3.48 & 14000 & 3.25 \\
\hline 7 & 12000 & 2.78 & 13000 & 3.02 & 14000 & 3.25 & 14000 & 3.25 \\
\hline 8 & 51000 & 11.83 & 23000 & 5.34 & 23000 & 5.34 & 23000 & 5.34 \\
\hline 9 & 33000 & 7.66 & 18000 & 4.18 & 18000 & 4.18 & 18000 & 4.18 \\
\hline 10 & 9000 & 2.09 & 13000 & 3.02 & 14000 & 3.25 & 13000 & 3.02 \\
\hline 11 & 8000 & 1.86 & 14000 & 3.25 & 13000 & 3.02 & 13000 & 3.02 \\
\hline 12 & 8000 & 1.86 & 13000 & 3.02 & 13000 & 3.02 & 13000 & 3.02 \\
\hline 13 & 7000 & 1.62 & 14000 & 3.25 & 14000 & 3.25 & 14000 & 3.25 \\
\hline 14 & 8000 & 1.86 & 13000 & 3.02 & 13000 & 3.02 & 13000 & 3.02 \\
\hline 15 & 9000 & 2.09 & 14000 & 3.25 & 13000 & 3.02 & 13000 & 3.02 \\
\hline 16 & 11000 & 2.55 & 16000 & 3.71 & 15000 & 3.48 & 15000 & 3.48 \\
\hline 17 & 18000 & 4.18 & 23000 & 5.34 & 21000 & 4.87 & 21000 & 4.87 \\
\hline 18 & 11000 & 2.55 & 13000 & 3.02 & 12000 & 2.78 & 12000 & 2.78 \\
\hline 19 & 10000 & 2.32 & 14000 & 3.25 & 10000 & 2.32 & 9000 & 2.09 \\
\hline 20 & 45000 & 10.44 & 14000 & 3.25 & 9000 & 2.09 & 10000 & 2.32 \\
\hline 21 & 8800 & 2.04 & 13000 & 3.02 & 9000 & 2.09 & 9000 & 2.09 \\
\hline 22 & 8700 & 2.02 & 13000 & 3.02 & 9000 & 2.09 & 11000 & 2.55 \\
\hline 23 & 8100 & 1.88 & 13800 & 3.20 & 11000 & 2.55 & 9000 & 2.09 \\
\hline 24 & 7500 & 1.74 & 12900 & 2.99 & 9000 & 2.09 & 9000 & 2.09 \\
\hline 25 & 52500 & 12.18 & 22100 & 5.13 & 68900 & 15.99 & 69200 & 16.06 \\
\hline 26 & 6800 & 1.58 & 20500 & 4.76 & 9600 & 2.23 & 9900 & 2.30 \\
\hline 27 & 6690 & 1.55 & 15800 & 3.67 & 10500 & 2.44 & 10500 & 2.44 \\
\hline 28 & 6822 & 1.58 & 18742 & 4.35 & 11739 & 2.72 & 11116 & 2.58 \\
\hline
\end{tabular}

La figura 9 indica que en la configuración geométrica de la torre de transmisión utilizada en este estudio para una línea de 500 kV no se genera una distribución de voltajes uniforme a lo largo de sus cadenas de aisladores. Pueden existir aisladores que lleguen a soportar voltajes excesivos, como en el caso del aislador número 25 , lo cual significaría un posible esfuerzo dieléctrico para ese aislador que con el paso del tiempo puede llegar a deteriorarse y producir 
una falla de aislamiento por contorneo del arco eléctrico o por perforación del material aislante del aislador.

Si bien el estudio realizado en este artículo no pudo ser contrastado con mediciones de campo, debido a las limitaciones de medición de niveles de voltaje tan elevados en las cadenas de aisladores en el caso de Ecuador, los resultados coinciden con las referencias presentadas en la introducción del artículo, que describen que la distribución de voltajes en las torres de transmisión no es uniforme y que se puede sugerir utilizar anillos metálicos en el primer aislador, o alargar la cadena de aisladores para mejorar la uniformidad de la distribución de voltajes. Con el método de trabajo presentado en el presente artículo, es posible profundizar este tipo de estudios adicionales, para mitigar el efecto negativo de esta distribución de voltajes y prevenir una falla de aislamiento en el futuro en la línea de transmisión.

Figura 9. (a) Distribución del voltaje en los aisladores de la cadena de aisladores tipo I. Lado derecho.

(b) Distribución del voltaje en los aisladores de la cadena de aisladores tipo I. Lado izquierdo.

(c) Distribución del voltaje en los aisladores del brazo derecho la cadena de aisladores tipo V. (d) Distribución del voltaje en los aisladores del brazo izquierdo la cadena de aisladores tipo $\mathrm{V}$

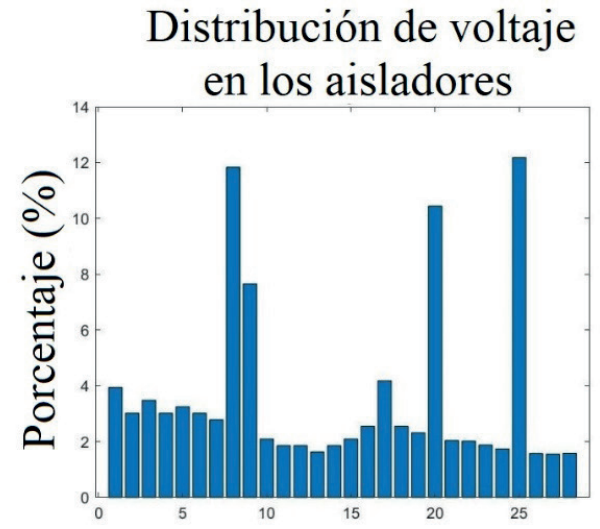

Nùmero de aislador

(a)

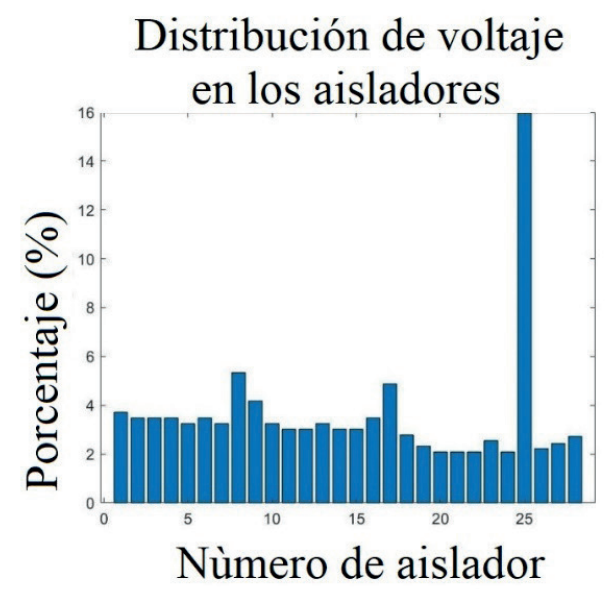

(c)

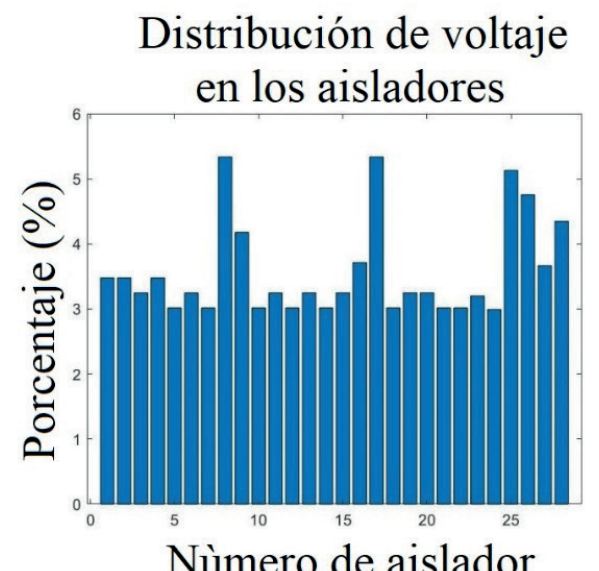

(b)

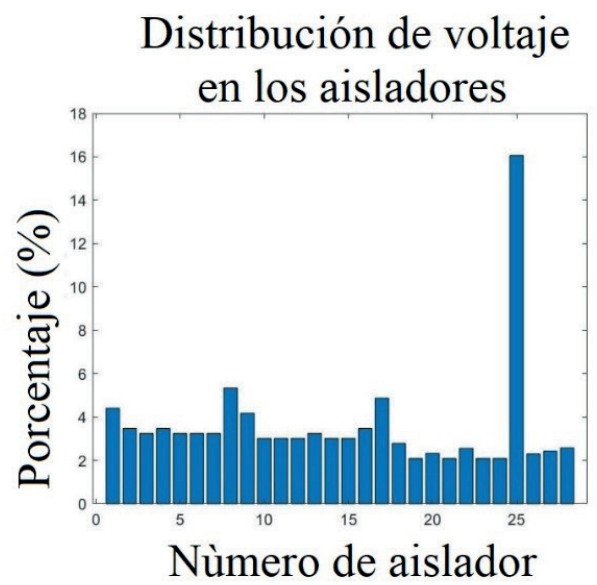

(d) 


\section{Conclusiones y recomendaciones}

Se ha presentado la implementación de un modelo FEM, de una línea de transmisión de 500 kV de Ecuador, con el fin de determinar la distribución de voltajes en su cadena de aisladores. Y se ha obtenido resultados que demuestran la flexibilidad de los métodos FEM, para la resolución de problemas electrostáticos asociados al alto voltaje y que pueden utilizarse como una guía en el diseño del aislamiento de las torres de transmisión de energía eléctrica, ya que es posible observar los lugares dentro de la cadena de aisladores, donde el voltaje es mayor, y modificar los diseños para tener una distribución de voltajes más uniforme; para ello se puede seguir el método descrito en este artículo.

Se observa que las distribuciones de voltaje en los aisladores no son uniformes, esto puede deberse a la geometría y los materiales utilizados en la simulación, lo cual afectaría a la distribución del campo eléctrico en el espacio en estudio. En general, se puede apreciar que la mayoría de los aisladores de la cadena soportan menos del $4 \%$ (aproximadamente $17 \mathrm{kV}$ ) del voltaje total de la cadena y por ello no corren ningún riesgo dieléctrico, pues están soportando un voltaje mucho menor a su nivel de aislamiento, que es de $90 \mathrm{kV}$; pero algunos aisladores pueden soportar mucho más voltaje hasta llegar a un máximo del 16.06 \% (aproximadamente $69.2 \mathrm{kV}$ ), en el aislador 25 de una de las cadenas de aisladores. Este voltaje es mayor al nivel de aislamiento en mojado del aislador, que es de $55 \mathrm{kV}$, por lo que en condiciones de lluvia podría presentarse una falla de aislamiento en ese aislador.

Los resultados presentados en este artículo pueden utilizarse para entender el efecto del campo eléctrico sobre el aislamiento de las torres de transmisión de energía eléctrica y se puede extender el estudio de este tema en las torres de transmisión de otros niveles de voltaje del sistema eléctrico ecuatoriano, para conocer la influencia del cambio de materiales en los aisladores y con diferentes geometrías en las torres de transmisión.

El modelo implementado puede utilizarse para realizar estimaciones de voltajes y campos eléctricos en todo el espacio de simulación y determinar posibles optimizaciones en sus diseños, al realizar modificaciones en los materiales y geometrías, que luego permitan observar cambios en las distribuciones de voltajes, y los campos eléctricos en los aislamientos y cercanías de una torre de transmisión.

\section{Bibliografía}

Akbari, E.; Mirzaie, M.; Rahimnejad, A. y Asadpoor, M. (2012). Finite Element Analysis of Disc Insulator Type and Corona Ring Effect on Electric Field Distribution over 230-kV Insulator Strings. International Journal of Engineering and Technology, 407-419.

Antonov, A. S.; Glushkov, D. A. y Kropotuhin, S. Y. (2016). A Set of Models for Investigation of Voltage Distribution along Suspension Insulator String. 2016 IEEE NW Russia Young Researchers in Electrical and Electronic Engineering Conference (EIConRusNW) (pp. 519-524). St. Petersburg, Russia: IEEE.

Arconel. (2013). Plan maestro de electrificación 2013-2022. Quito-Ecuador: Arconel.

Arora, R. y Mosch, W. (2011). High Voltage Insulation Engineering. Institute of Electrical and Electronics Engineers.

Ashouri, M.; Mirzaie, M. y Gholami, A. (2010). Calculation of Voltage Distribution along Porcelain Suspension Insulators Based on Finite Element Method. Electric Power Components and Systems, 820-831.

Benguesmia, H.; M'Ziou, N. y Boubakeur, A. (2018). Simulation of the Potential and Electric Field Distribution on High Voltage Insulator using the Finite Element Method. Diagnostyka, 19(2), 41-52. 
Bessedik, S. (2015). Contournement des isolateurs pollués. Algeria: Ph.D. thesis in Sciences, Department of Electrical Engineering, University of Oran.

Chen, L. y Liang, X. (2012). Computational Analysis on Voltage Distribution along Ceramic Insulator Strings of Uhv Ac Transmission Line. Gaodianya Jishu/ High Voltage Engineering, 376-381.

Conelec. (2013). Perspectiva y expansión del sistema eléctrico ecuatoriano. Quito: Conelec. Recuperado de https://www.regulacionelectrica.gob.ec/wp-content/uploads/downloads/2015/12/Vol3Perspectiva-y-expansi\%C3\%B3n-del-sistema-el\%C3\%A9ctrico-ecuatoriano.pdf

Du Zhiye, L. T. y Daochun, H. (2010). Parallel Computation of Voltage Distribution Along Composite Insulator Strings and Electric Field Intensity on the Surface of Hardware Fittings. Transactions of China Electrotechnical Society.

Huang, D. C.; Ruan, J. J. y Liu, S. B. (2010). Potential Distribution along UHV AC transmission Line Composite Insulator and Electric Field Distribution on the Surface of Grading Ring. Gaodianya Jishu/ High Voltage Engineering, 1442-1447.

Khare, T.; Hasabe, R. y Mandlik, M. (2016). Electric Field and Potential Distribution along Porcelain Insulator under Polluted Conditions using Finite Element Method. International Journal of Advanced Research in Electrical, Electronics and Instrumentation Engineering, 5(5), 3531-3536.

Meeker, D. (2006). Finite Element Method Magnetics User's Manual Version 4.2.

Meeker, D. (2018). Finite Element Method Magnetics: pyFEMM User's Manual.

Muniraj, C. y Chandrasekar, S. (2012). Finite Element Modeling for Electric Field and Voltage Distribution along the Polluted Polymeric Insulator. World Journal of Modelling and Simulation, 8(4), 310-320.

Nicolopoulou, E.; Gralista, E.; Kontargyri, V. et al. (2011). Electric Field and Voltage Distribution around Composite Insulators. XVII International Symposium on High Voltage Engineering. Hannover.

Ordal, M.; Long, L.; Bell, R. et al. (1982). Optical Properties of the Metals Al, Co, Cu, Au, Fe, Pb, Ni, Pd, Pt, Ag, $\mathrm{Ti}$, and W in the Infrared and Far Infrared. Applied Optic, 22(7), 1099-1120.

Radwan, R.; Mahdy, A.; Abdel-Salam, M. y Samy, M. (2013). Electric Field Mitigation under Extra High Voltage Power Lines. IEEE Transactions on Dielectrics and Electrical Insulation, 54-62.

Sima, W.; Yuan, T.; Yang, Q. et al. (2010). Effect of Non-uniform Pollution on the Withstand Characteristics of Extra High Voltage (EHV) Suspension Ceramic Insulator String. IET Generation, Transmission \& Distribution, 445-455.

Transelectric, C. E. (2015). El Sistema de transmisión de 500 kV contribuye al desarrollo energético del país. Recuperado de: https://www.celec.gob.ec/78-quienes-somos/482-el-sistema-de-transmision-de-500-kv-contribuye-al-desarrollo-energetico-del-pais.html

Transeselectric, C. E. (2013). Estudio de impacto ambiental definitivo. Sistema de transmisión de extra alta tensión y sistemas asociados. 50. Quito.

Wadhwa, C. L. (1989). Generation, Distribution and Utilization of Electrical Energy. United States of America: John Wiley and Sons.

Zhang, B.; He, J.; Zeng, R. y Liang, X. (2010). Voltage Distribution along a Long Ceramic Insulator String in a High-Voltage Tower Window. COMPEL-The international journal for computation and mathematics in electrical and electronic engineering., 811-823. 